OAK RIDGE NATIONAL LABORATORY

\section{MARTIN MARIETRA}

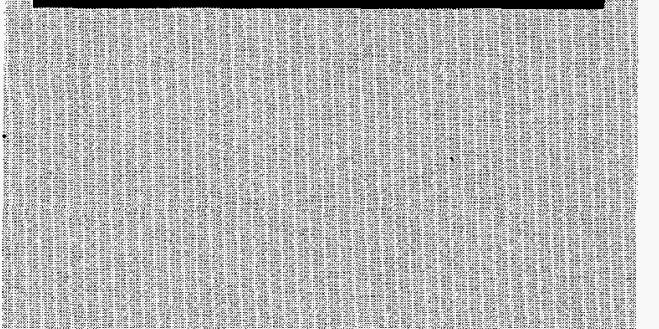

(1)
New Insights into Input Relegation Control for Inverse Kinematics of a Redundant Manipulator Part 1: On the Orthogonality of Matrices $B$ and $J$ and Comparison to the Extended Jacobian Method
M. A. Unseren
D. B. Reister 
This report has been reproduced directly from the best available copy.

Available to DOE and DOE contractors from the Office of Scientific and Technical Information, P.O. Box 62, Oak Ridge, TN 37831; prices available from (615) 576-8401. FTS 626-8401.

Available to the public from the National Technical Information Service, U.S. Department of Commerce, 5285 Port Royal Rd., Springfield, VA 22161.

NTIS price codes-Printed Copy: AO3 Microfiche AO1

This report was prepared as an account of work sponsored by an agency of the United States Government. Neither the United States Government nor any agency thereof. nor any of their employees, makes any warranty, express or implied, or assumes any legal liability or responsibility for the accuracy, completeness, or usefulness of any information, apparatus, product, or process disclosed, or represents that its use would not infringe privately owned rights. Relerence herein to any specific commercial product, process, or service by trade name, trademark. manufacturer, or otherwise, does not necessarily constilute or imply its endorsement, recommendation, or lavoring by the United States Government or any agency thereot. The views and opinions of authors expressed herein do not necessarily state or reflect those of the United States Government or any agency thereof. 


\section{DISCLAIMER}

Portions of this document may be illegible in electronic image products. Images are produced from the best available original document. 
ORNL/TM-12813 (Part 1)

Computer Science and Mathematics Division

\title{
NEW INSIGHTS INTO INPUT RELEGATION CONTROL FOR INVERSE KINEMATICS OF A REDUNDANT MANIPULATOR \\ PART 1: ON THE ORTHOGONALITY OF MATRICES $B$ AND $J$ AND \\ COMPARISON TO THE EXTENDED JACOBIAN METHOD
}

\author{
M. A. Unseren and D. B. Reister \\ Center for Engineering Systems Advanced Research
}

DATE PUBLISHED: JULY, 1995

Prepared by

Oak Ridge National Laboratory

Oak Ridge, Tennessee 37831

managed by

LOCKHEED MARTIN ENERGY SYSTEMS, INC.

for the

U. S. DEPARTMENT OF ENERGY

under Contract No. DE-AC05-84OR21400

DISTRIBUTION OF THIS DOCUMENT IS UNLIMITED 


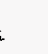




\section{CONTENTS}

Abstract................................................ v

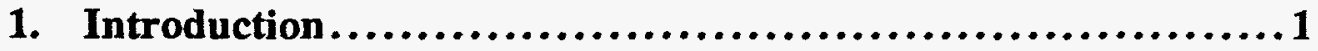

2. New Insights in Choosing B Orthogonal to $\mathrm{J} \ldots \ldots \ldots \ldots \ldots \ldots$

3. Comparison to Related Works $\ldots \ldots \ldots \ldots \ldots \ldots \ldots \ldots \ldots \ldots \ldots$

4. Conclusion..$\ldots \ldots \ldots \ldots \ldots \ldots \ldots \ldots \ldots \ldots \ldots \ldots \ldots \ldots \ldots \ldots \ldots \ldots$

5. Acknowledgement $\ldots \ldots \ldots \ldots \ldots \ldots \ldots \ldots \ldots \ldots \ldots \ldots \ldots \ldots \ldots \ldots$

6. References....................................... 15 


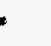

$$
\text { - }
$$




\begin{abstract}
A method for kinematically modeling a constrained rigid body mechanical system $[1,2]$ and a method for controlling such a system termed input relegation control (IRC) [3] were applied to resolve the kinematic redundancy of a serial link manipulator moving in an open chain configuration in [6]. A set of equations was introduced to define a new vector variable parameterizing the redundant degrees of freedom (DOF) as a linear function of the joint velocities [6]. The new set was combined with the classical kinematic velocity model of a manipulator and solved to yield a well specified solution for the joint velocities as a function of the Cartesian velocities of the end effector and of the redundant DOF variable. In the previous work a technique was proposed for selecting the matrix relating the redundant DOF variable to the joint velocities which resulted in it rows being orthogonal to the rows of the Jacobian matrix. The implications for such a selection were not discussed in [6]. In Part 1 of this report a basis for the joint space is suggested which provides considerable insight into why picking the aforementioned matrix to be orthogonal to the Jacobian is advantageous. A second objective of Part 1 is to compare the IRC method to the Extended Jacobian method of Baillieul and Martin [13, 14] and other related methods.
\end{abstract}




\section{Introduction}

The redundancy resolution scheme presented in this report is based on a method for kinematically modeling a constrained rigid body mechanical system $[1,2]$ and on a theory for controlling such a system termed input relegation control (IRC) [3]. The mechanical system considered in $[1,2]$ has $n$ actuators whose $n$ generalized velocities must satisfy a set of $k$ integrable or nonintegrable linear bilateral constraints $(k<n)$. Such a constrained system has $(n-k)$ position degrees of freedom (DOF). It is easy to see that there are infinitely many solutions for the generalized velocities which satisfy the constraints. A new set of $(n-k)$ independent generalized velocities, termed pseudovelocities, was introduced and defined as a linear function of the coupled generalized velocities. These equations, when combined with the constraints, may be solved to yield a single, well specified solution for the generalized velocities $[1,2]$. The theory of IRC suggests that several tasks can be accomplished simultaneously when controlling the constrained system [3]. One task would be to control the motion of the system while satisfying the constraints. The additional task would be to control the Lagrangian constraint forces which arise due to the loss of the position DOF. Control laws are designed for each task, and the control inputs to the actuators are comprised of the sum of the task controllers (or transformations of them).

A classical example of a control law based on the principles of IRC was provided in Kankaanranta and Koivo [4]. It was suggested in [4] that the system's position DOF can be utilized to explicitly control the pseudovelocities and the pseudocoordinates (i.e., the time integrals of the pseudovelocities) to track reference trajectories. The loss of the $k$ position DOF gives rise to $k$ force DOF, and Kankaanranta et al. as well as others [5] have suggested that the force DOF can be used to explicitly control the system's $k$ independent Lagrangian constraint forces to track reference trajectories. The pseudovariable and Lagrangian constraint force task controllers were transformed into the joint space, which, when applied to the model, resulted in an explicit decoupling of the position- and force-controlled DOF [4].

To review how the kinematic modeling method $[1,2]$ and the concept of IRC [3] were first applied to the kinematic redundancy resolution during motion of a fixed base, open chain, serial link redundant manipulator in [6], it is convenient to present the classical kinematic velocity model for such a manipulator:

$$
\dot{x}=J \dot{q}
$$

where $\dot{x}=\left[\dot{x}_{1}, \dot{x}_{2}, \ldots, \dot{x}_{M}\right]^{T}$ is the vector of Cartesian velocities of the end effector with respect to the base coordinate system of the manipulator. $\dot{q}=\left[\dot{q}_{1}, \dot{q}_{2}, \ldots, \dot{q}_{N}\right]^{T}$ is the vector of joint velocities. Superscript $T$ denotes a matrix transposition and it is assumed that $M<N$. The $(M \times N)$ Jacobian matrix $J(q)$ in eq. (1) is assumed to have full rank $M$.

The columns of $J$ comprise $N$ vectors in the $M$ dimensional task (Cartesian) space. Only $M$ of these $N$ vectors comprise a linearly independent set which spans the task space. The rows of $J$ comprise a set of $M$ vectors in the $N$ dimensional joint space. These $M$ row vectors comprise a linearly independent set. However, $L=(N-M)$ additional row vectors are needed to comprise a linearly independent set which spans the joint space.

The original application of IRC to kinematic redundancy resolution [6] sought to define those $L$ additional row vectors. The idea was to introduce a new vector variable $\epsilon=\left[\epsilon_{1}, \epsilon_{2}, \ldots, \epsilon_{L}\right]^{T}$ which parameterizes the redundant DOF and define it as a linear combination of the joint velocities $\dot{q}$ : 


$$
\epsilon=B \dot{q}
$$

where the $L$ row vectors comprising matrix $B(q)$ are selected such that the composite $(N \times N)$ matrix $S(q)$, defined by:

$$
S=\left[\begin{array}{l}
J \\
B
\end{array}\right]
$$

is nonsingular. It is convenient to partition the inverse of $S$ into two matrices [6]:

$$
S^{-1}=\left[\begin{array}{ll}
E, & F
\end{array}\right]
$$

where $E(q)$ and $F(q)$ are $(N \times M)$ and $(N \times L)$ matrices, respectively. Eqs. (3) and (4) imply the following five matrix identities:

$$
\begin{gathered}
J E=I_{M \times M}, \\
J F=0_{M \times L}, \\
B E=0_{L \times M}, \\
B F=I_{L \times L}, \\
E J+F B=I_{N \times N} .
\end{gathered}
$$

Here $I_{k \times k}$ denotes a $(k \times k)$ identity matrix and $0_{k \times l}$ a $(k \times l)$ matrix of zeros.

Eqs. (1) and (2) can be combined and solved for the joint velocities:

$$
\dot{q}=E \dot{x}+F \epsilon .
$$

The first term $(E \dot{x})$ to the right of eq. (10) is the particular solution to eq. (1) which causes the end effector to physically move. The second term $(F \epsilon)$ is the homogeneous solution to eq. (1) which causes self motion of the joints (i.e., motion of the joints that does not induce end effector motion).

A designer whom uses IRC to resolve kinematic redundancy of a manipulator must address two major issues: (i) Selection of the matrices $\{B, E, F\}$ given $J$ and (ii) determining $\epsilon$. Only when these quantities are known can we compute $\dot{q}$ using eq. (10). The first issue is the subject of Part 1 of this report, whereas the problem of selecting $\epsilon$ to optimize a secondary criteria involving self motion of the joints is the subject of Parts 2 and $3[7,8]$.

In our previous work on the first issue, it was assumed that given $J, B$ is picked, which immediately leads to the determination of $\{E, F\}$ by eq. (4). An optimization technique was suggested for choosing $B$ for manipulators with a single degree of redundancy $(L=1)$. The choice resulted in maximizing the determinant of matrix $S$ where $B$ was restricted to be a normalized vector. It was discovered that the resulting $B$ was orthogonal to the rows of the Jacobian $J$. It was also shown that when the rows of $B$ and $J$ are orthogonal, the columns of $E$ and $F$ are also orthogonal. However, the implications and advantages of having $B$ orthogonal to $J$ were not discussed in [6].

The $L$ column vectors comprising matrix $F$ in eq. (4) all lie in the null space of the Jacobian matrix $J$. They form a basis for the null space of $J$ and are immediately determined when $B$ is selected such that $S$ is nonsingular. A critical view of the earlier work reveals that the idea of first choosing $B$ then determining $F$ (and $E$ ) is somewhat illogical. Indeed, it implies that the rows of $S$ are a suitable set of basis vectors which span the $N$ dimensional joint space. However, the rows of $B$ may or may not be orthogonal to the rows of $J$. It is obvious that the former case is the 
most desirable because orthogonality is the strongest form of linear independence of a pair of vectors [9]. In section 2 we re-examine the problem of determining matrix $B$ which provides intuitive insight into the benefits of choosing $B$ to be orthogonal to $J$.

Past experience indicates that there is some confusion about differences between the IRC and Extended Jacobian methods [13,14] of kinematic redundancy resolution. In section 3 the two methods are compared based on a mathematical review of the Extended Jacobian method. The IRC method is also compared to some other approaches. 


\section{New Insights in Choosing $B$ Orthogonal to $J$}

In this section we take an alternative view about which of the four matrices $\{J, B, E, F\}$ are known apriori and which are unknown. For the purposes of this report, it is assumed that $J$ and $F$ are known apriori and the problem is to solve for $B$ and $E$ in terms of the known matrices, while satisfying eqs. (5) - (9). There exist numerical methods for determining a basis for the null space of a rectangular matrix with fewer rows than columns, e.g., the zero eigenvalue matrix theorem $[10,11]$. Some analytical techniques for determining such a basis also exist $[5,6,12]$. Therefore the assumption that $F$ is known apriori is both logical and reasonable. Insight into the advantages of choosing $B$ orthogonal to $J$ will be much more clear using this approach.

Let the columns of the $(N \times N)$ matrix $G$, defined by:

$$
G=\left[J^{T}, \quad F\right]
$$

be the basis for the $N$ dimensional joint space.

The basis defined in eq. (11) can provide insight into the problem of determining $B$ and $E$ such that eqs. (5) - (9) are satisfied. Expressing the $k$ th row of $B$ in terms of the transposed basis vectors gives:

$$
B_{k}=\sum_{i=1}^{M} \alpha_{k i} J_{i}+\sum_{j=1}^{L} \gamma_{k j} F_{j}^{T} ; k=1,2, \ldots, L
$$

where $J_{i}$ is the ith row of $J$ and $F_{j}$ is the $j$ th column of $F$. The $k$ th column of $E$ can be defined in a similar manner:

$$
E_{k}=\sum_{i=1}^{M} J_{i}^{T} \zeta_{i k}+\sum_{j=1}^{L} F_{j} \varepsilon_{j k} ; \quad k=1,2, \ldots, M .
$$

It is convenient to express eqs. (12) and (13) as concise matrix equations:

$$
\begin{gathered}
B=\alpha J+\gamma F^{T}, \\
E=J^{T} \zeta+F \varepsilon
\end{gathered}
$$

where $\alpha$ and $\varepsilon$ are $(L \times M)$ matrices, $\gamma$ is an $(L \times L)$ matrix, and $\zeta$ is an $(M \times M)$ matrix.

Postmultiplying eq. (14) by $[E, F]$ and invoking eqs. (7) and (8) give:

$$
\left[\alpha+\gamma F^{T} E, \quad \gamma F^{T} F\right]=\left[\begin{array}{ll}
0_{L \times M}, & I_{L \times L}
\end{array}\right]
$$

The symbolic solutions for $\gamma$ and $\alpha$ based on eq. (16) are:

$$
\begin{gathered}
\gamma=\left(F^{T} F\right)^{-1} \\
\alpha=-\left(F^{T} F\right)^{-1} F^{T} E .
\end{gathered}
$$

Premultiplying eq. (15) by matrix $S$ and invoking eqs. (3),(5), and (7) give:

$$
\left[\begin{array}{c}
J J^{T} \zeta \\
B J^{T} \zeta+\varepsilon
\end{array}\right]=\left[\begin{array}{c}
I_{M \times M} \\
0_{L \times M}
\end{array}\right]
$$


The symbolic solutions for $\zeta$ and $\varepsilon$ based on eq. (19) are given by:

$$
\begin{gathered}
\zeta=\left(J J^{T}\right)^{-1}, \\
\varepsilon=-B J^{T}\left(J J^{T}\right)^{-1} .
\end{gathered}
$$

Replacing $E$ in eq. (18) by the right hand side of eq. (15) yields:

$$
\varepsilon=-\alpha \text {. }
$$

The identical result can be obtained by replacing $B$ in eq. (21) by the right hand side of eq. (14).

The expressions for $B$ and $E$ can now be simplified:

$$
\begin{gathered}
B=\alpha J+\left(F^{T} F\right)^{-1} F^{T}, \\
E=J^{T}\left(J J^{T}\right)^{-1}-F \alpha .
\end{gathered}
$$

It is repeated for emphasis that the term $(E \dot{x})$ in eq. (10) is the particular solution to eq. (1). However, when $E$ is defined by eq. (24), the term $(E \dot{x})$ contains a component $\{-F \alpha \dot{x}\}$ that lies in the null space of $J$ and thus induces self motion of the joints. That is to say, the self motion component of solution in eq. (10) is $\{F(\epsilon-\alpha \dot{x})\}$ and not $(F \epsilon)$ as we might expect. The point being is that vector $\epsilon$, defined by eq. (2), does not completely parameterize the redundant DOF in the solution for the joint velocities given by eq. (10) as long as $-F \alpha \dot{x} \neq 0_{N \times 1}$. Furthermore, the self motions induced by $\{F \epsilon\}$ and $\{-F \alpha \dot{x}\}$ might conflict with one another when determining a solution for the joint velocities that satisfies a secondary objective using self motion of the joints, the topic of Part 2.

To prevent such a potential conflict, a particular solution to eq. (1) is sought which does not induce self motion of the joints. This will occur when the parameter matrix $\alpha=0_{L \times M}$ in eq. (24). Noting eqs. (21) and (22), $\alpha$ becomes a matrix of zeros when the rows of $B$ are selected to lie in the null space of $J$, i.e.:

$$
J B^{T}=0_{M \times L} .
$$

When eq. (25) applies, the solution for the joint velocities expressed in terms of the joint space basis $G$ is:

$$
\dot{q}=G\left[\begin{array}{c}
\left(J J^{T}\right)^{-1} \dot{x} \\
\epsilon
\end{array}\right]
$$

At this point, eq. (26) is only an intermediate solution for $\dot{q}$ because $\epsilon$ is still unknown. Further motivation for choosing $B$ orthogonal to $J$ is discussed in section 3 of Part 2 of this report, where $\epsilon$ is determined by optimization techniques to yield the final solution for $\dot{q}$. 


\section{Comparison to Related Works}

The described input relegation control redundancy resolution scheme is compared to the Extended Jacobian method of Baillieul and Martin [13,14] and other related literature in this section. In Baillieul et al., $[13,14]$ a set of equations of the form of eq. (2) was introduced with the restriction that $\epsilon=0_{L \times 1}$, where, here again, $L=N-M$. The solution for $\dot{q}$ suggested in $[13,14]$ is:

$$
\dot{q}=S^{-1}\left[\begin{array}{c}
\dot{x} \\
0_{L \times 1}
\end{array}\right]
$$

where the square matrix $S$ defined in eq. (3) was referred to as the Extended Jacobian matrix.

It is convenient to apply the partitioned inverse defined by eq. (4) to simplify eq. (27):

$$
\dot{q}=E \dot{x} .
$$

Thus the Extended Jacobian method yields only a particular solution to eq. (1) whereas the IRC method yields both particular and homogeneous solutions to eq. (1).

In Baillieul et al. [13,14], it was assumed that the null space $F$ of $J$ is known a priori. The $i$ th row of $B$ was determined by the following procedure: (i) the values of the joint positions $q$ are determined such that the Lie derivative of a scalar performance criteria $h(q)$ in the direction of the $i$ th column of $F$ equates to zero:

$$
g_{i}=\frac{\partial h}{\partial q} F_{i}=0
$$

where $g_{i}(q)$ is the scalar Lie derivative of $h, F_{i}$ signifies the $i$ th column of $F$, and $i=1,2, \ldots, L$. (ii) the partial derivative of $g_{i}$ with respect to $q$ is obtained:

$$
B_{i}=\frac{\partial g_{i}}{\partial q}
$$

where $B_{i}$ signifies the $i$ th row of $B$. The components of the $i$ th row of $B$ are calculated using the values of $q$ which result in $g_{i}=0$.

Scalar $g_{i}$ could be a highly nonlinear transcendental function of the joint positions. An analytical procedure for determining the values of $q$ such that $g_{i}=0$ was not discussed in Baillieul et al. $[13,14]$. It is true that an example was given in [13, 14] where $q$ was determined such that $g_{i}=0$ for a simplistic configuration of a three link planar revolute manipulator, but even then the link lengths were all set to unity. However, the problem of determining $q$ such that eq. (29) is satisfied for a general spatial multilink redundant manipulator must be accomplished by numerical techniques.

The symbolic representation of the inverse of $S$ was obtained by the following procedure in [13]:

$$
\left[\begin{array}{l}
J \\
B
\end{array}\right]\left[J^{T}\left(J J^{T}\right)^{-1}, \quad F\right]=\left[\begin{array}{cc}
I_{M \times M} & 0_{M \times L} \\
B J^{T}\left(J J^{T}\right)^{-1} & I_{L \times L}
\end{array}\right] .
$$

Then, by a mathematical observation: 


$$
\left[\begin{array}{c}
J \\
B
\end{array}\right]^{-1}=\left[J^{T}\left(J J^{T}\right)^{-1}, F\right]\left[\begin{array}{cc}
I_{M \times M} & 0_{M \times L} \\
-B J^{T}\left(J J^{T}\right)^{-1} & I_{L \times L}
\end{array}\right] .
$$

Noting eq. (4), the solution for $E$ required to solve for $\dot{q}$ in eq. (28) is obtained from eq. (32) and may be expressed in terms of the joint space basis $G$ that was defined in eq. (11):

$$
E=G\left[\begin{array}{c}
I_{M \times M} \\
-B J^{T}
\end{array}\right]\left(J J^{T}\right)^{-1}
$$

It should be mentioned that the symbolic solution for $E$ in eq. (33) can be obtained by postmultiplying eq. (9) by $J^{T}\left(J J^{T}\right)^{-1}$ and rearranging terms.

By substituting eq. (33) into eq. (28), the solution for the joint velocities presented in $[13,14]$ is expressed in terms of $G$ :

$$
\dot{q}=G\left[\begin{array}{c}
I_{M \times M} \\
-B J^{T}
\end{array}\right]\left(J J^{T}\right)^{-1} \dot{x}
$$

Please note that the particular solution to eq. (1) given by eq. (34) contains a component which induces self motion of the joints. When $B$ is orthogonal to $J$, the solution obtained by eq. (34) is identical to Whitney's pseudoinverse solution [15] for the joint velocities.

It is repeated for emphasis that the solution for $\dot{q}$ obtained by IRC would contain the additional term $(F \epsilon)$ on the right hand side of eq. (34).

In the Configuration Control method [16], a set of $L$ user defined "self motion" kinematic functions $\Phi=\left[\phi_{1}, \phi_{2}, \ldots, \phi_{L}\right]^{T}$ are defined as functions of the joint variables, i.e., $\Phi=\Phi(q)$. These functions along with the end effector Cartesian coordinates formed a set of $N$ task-related configuration variables. Reference trajectories were specified for the task-related configuration variables, and adaptive controllers were devised to control these variables to track the reference trajectories.

Please note that the quantities $\dot{\Phi}$ and $J_{c}$ in [16] correspond to the quantities $\epsilon$ and $B$ used here, respectively. In our approach $\epsilon$ is an unknown quantity whose value is determined as part of the inverse kinematics optimization process. One the other hand, the Configuration Control method does not involve inverse kinematics.

A general solution to eq. (1) was proposed in [17] which can be expressed using the notation of this paper:

$$
\dot{q}=\left[\begin{array}{c}
J \\
F^{T}
\end{array}\right]^{-1}\left[\begin{array}{c}
\dot{x} \\
F^{T} F \epsilon
\end{array}\right] .
$$

In [17], it was claimed that $F$ can be determined analytically such that eq. (6) is satisfied by solving the determinant equation $\left|J J^{T}\right|=\left|F^{T} F\right|$. It was acknowledged in [17] that the method involves some guesswork. In the example given, the symbolic $(6 \times 7)$ Jacobian $J$ had a simple structure: 24 of its elements were zero valued, four of its elements were equal to one, and the remainder were functions of the joint angles. It appeared that the simplicity of their $J$ was instrumental in being able to solve for the 7 dimensional vector $F$. The Jacobian matrix considered in this paper transforms the joint velocities to obtain the Cartesian velocities of the end effector expressed in the base coordinate system. The symbolic representation of this matrix 
is considerably more complex than the one illustrated in [17] and it may be difficult or even impossible to solve the determinant equation for $F$.

An application of the previous work [4] to resolve the redundancy during open chain motion of a 3-link planar revolute manipulator is discussed in [18] where only the Cartesian translational motion of the end effector is specified. The procedure suggested in [18] is equivalent to selecting $\epsilon$ to be the Cartesian angular velocity of the end effector with $B=[1,1,1]$. But given these choices for $\{\epsilon, B\}$, eq. (2) would in fact comprise the third row of the kinematic velocity model in eq. (1) using the approach given here. Furthermore, specifying the Cartesian translational and rotational motions of the end effector of a 3-link planar revolute manipulator does not constitute a kinematically redundant system. 


\section{Conclusion}

To better understand the advantages of choosing $B$ orthogonal to the Jacobian $J$ when resolving the kinematic redundancy of a serial link, open chain manipulator using the input relegation control (IRC) method as suggested in [6], a proper basis for the $N$ dimensional joint space is needed. In this report it was suggested that the proper basis consists of the rows of $J$ along with the vectors comprising its null space (i.e., the columns of $F$ ). It was assumed that the basis vectors are known, and the unknown matrices $\{B, E\}$ were expressed as linear combinations of the basis vectors.

This formulation proved to be insightful in choosing a specific solution for $B$ and $E$. Indeed, it was noticed that when $E$ has a component in the null space of $J$, the particular solution to the original kinematic velocity model, namely $(E \dot{x})$, contains a component which induces self motion of the joints. This is in addition to the self motion induced by the homogeneous solution to the original system, namely $(F \epsilon)$. In this situation it can be argued that vector $\epsilon$ does not completely parameterize the redundant degrees of freedom. Moreover, the self motions induced by the particular and homogeneous solutions might conflict with one another. To prevent such a potential conflict, it was suggested to select $B$ orthogonal to $J$, which also results in $E$ being orthogonal to $F$. Now there is no self motion component in the particular solution.

The report also clarified the distinctions between input relegation control and the Extended Jacobian method of Baillieul and Martin [13, 14]. The fundamental differences are: (i) the Extended Jacobian method yields only a particular solution for the joint velocities based on the kinematic velocity model, whereas the IRC method yields a particular and a homogeneous solution to the model. Mathematically, the Extended Jacobian symbolic solution is obtained by restricting the redundant degree of freedom quantifying variable $\epsilon$ introduced here to be an $(L \times 1)$ vector of zeros.; (ii) In IRC, $B$ is selected to be orthogonal to $J$ such that the particular solution for the joint velocities contains no component inducing self motion whereas in general the particular solution obtained by the Extended Jacobian method contributes to end effector motion and self motion. The methods determine $B$ and $E$ differently. 
, . 


\section{Acknowledgement}

This research was sponsored by the Office of Engineering Research Program, Basic Energy Sciences, of the U.S. Department of Energy, under contract DE-AC05$840 R 21400$ with Lockheed Martin Energy Systems, Inc. 


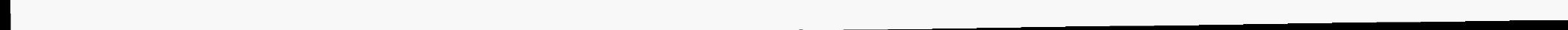




\section{References}

[1] F.R. Gantmacher, Lectures in Analytical Mechanics, USSR: Mir Publishers, 1975, chap. 1 , sec.10.

[2] H. Hemami, "A Feedback On-Off Model of Biped Dynamics," IEEE Trans. on Systems, Man, and Cybernetics vol. SMC-10, No. 7, pp. 376-383, July 1980, Sec. IV.

[3] H. Hemami and H.D. Ong, "Philosophy, Structure, and Examples of Relegated Control," J. of Intelligent and Robotic Systems Vol. 2, pp. 53-72, 1989.

[4] R.K. Kankaanranta and H.N. Koivo, "Dynamics and Simulation of Compliant Motion of a Manipulator," IEEE J. Robotics and Automation vol. 4, no. 2, pp. 163-173, April 1988.

[5] H. Hemami, C. Wongchaisuwat, and J.L. Brinker, "A Heuristic Study of Relegation of Control in Constrained Robotic Systems," ASME J. of Dynamic Systems, Measurement, and Control Vol. 109, pp. 224-231, September 1987.

[6] M.A. Unseren, "Input Relegation Control for Gross Motion of a Kinematically Redundant Manipulator," Oak Ridge National Laboratory Technical Report No. ORNL/TM-12165, October 1992.

[7] M.A. Unseren, "New Insights Into Input Relegation Control for Inverse Kinematics of a Redundant Manipulator. Part 2: The Optimization of a Secondary Criteria Involving Self Motion of the Joints," Oak Ridge National Laboratory Technical Report No. ORNL/TM-12813 (Part 2), June 1995.

[8] M.A. Unseren, "New Insights Into Input Relegation Control for Inverse Kinematics of a Redundant Manipulator. Part 3: An Application to Joint Limit Avoidance," Oak Ridge National Laboratory Technical Report No. ORNL/TM12813 (Part 3), June 1995.

[9] B. Noble and J.W. Daniel, Applied Linear Algebra, Prentice-Hall Publisher, second ed., 1977.

[10] W.C. Walton, Jr. and E.C. Steeves, "A New Matrix Theorem and Its Application for Establishing Independent Coordinates for Complex Dynamical Systems With Constraints," NASA Technical Report TR R-326, 1969.

[11] J.W. Kamman and R.L. Huston, "Dynamics of Constrained Multibody Systems," ASME J. of Applied Mechanics vol. 51, pp. 899-903, December 1984.

[12] D.B. Reister and M.A. Unseren, "Position and Force Control of a Vehicle with Two or More Steerable Drive Wheels," IEEE Trans. on Robotics and Automation vol. 9, no. 6, pp. 723-732, December 1993.

[13] J. Baillieul, "Kinematic Programming Alternatives for Redundant Manipulators," IEEE Int'l Conf. Robotics and Automation St. Louis, Mo., March 25-28, 1985, pp. 722-728.

[14] J. Baillieul and D.P. Martin, "Resolution of Kinematic Redundancy" in Robotics, Proceedings of Symposia in Applied Mathematics, Vol. 41, pp.49-90, Louisville, Kentucky, January 1990 (American Mathematical Society Short Course Lecture Notes). 
[15] D.E. Whitney, "Resolved motion rate control of manipulators and human prosthesis," IEEE Trans. on Man-Machine Systems, vol. 10, 1969, pp. 47-53.

[16] H. Seraji, "Configuration Control of Redundant Manipulators: Theory and Implementation," IEEE Trans. on Robotics and Automation vol. 5, no. 4, pp. 472490, August 1989.

[17] Y.L. Gu, "Dynamics and Control for Redundant Robots," IEEE Int'l Conf. on Robotics and Automation, Vol. 1, pp. 194-199, Philadelphia, PA, April 24-29, 1988.

[18] S.H. Arnautovic, "Constraint-Based Resolution and Control of Redundant Manipulators Via Pseudovariables," PhD Thesis, Purdue University, W. Lafayette, IN, August 1990. 
ORNL/TM-12813/Part 1

\section{INTERNAL DISTRIBUTION}

1-5. J. Barhen

6. M. Beckerman

7. C. W. Glover

8-12. W. C. Grimmell

13. J. F. Jansen

14. J. P. Jones

15. H. E. Knee

16. R. L. Kress

17. D. S. Kwon

18-22. R. C. Mann

23. M. D. Morris

24. E. M. Oblow

25-29. C. E. Oliver

30. L. E. Parker
31. F. G. Pin

32. N. S. V. Rao

33-37. D. B. Reister

38. S. Shekhar

39-43. R. F. Sincovec

44. D. Thompson

45. E. C. Uberbacher

46-50. M. A. Unseren

51. CSMD Reports Office

52-53. Laboratory Records Department

54. Laboratory Records, ORNL-RC

55. Document Reference Section

56. Central Research Library

57. ORNL Patent Office

\section{EXTERNAL DISTRIBUTION}

58. Dr. Fred Aminzadeh, 401 Paseo Estrella, Anaheim Hills, CA 92807

59. Dr. John S. Bay, Bradley Department of Electrical Engineering, Virginia Polytechnic Institute and State University, Blacksburg, VA 24061

60. Dr. John Blair, JBX Technologies, 25 Moore Road, Wayland, MA 01778

61. Professor Roger W. Brockett, Harvard University, Pierce Hall, 29 Oxford St., Cambridge, MA 02138

62. Dr. H. Hemami, Ohio State University, Department of Electrical Engineering, Columbus, Ohio 43210

63. Mr. Steve Holland, Robotics, B/MD-63, General Motors Corporation, NAO Manufacturing Center, 30300 Mound Rd., Warren, MI 48090-9040

64. Professor Takeo Kanade, Computer Science and Robotics, Carnegie Mellon University, Pittsburgh, PA 15213-3890 
65. Dr. Oscar P. Manley, Division of Engineering, Mathematical, and Geosciences, Office of Basic Energy Sciences, ER-15, U.S. Department of Energy, Germantown, Washington, DC 20545

66. Dr. K. S. Narendra, Yale University, Center for Systems Science, Department of Electrical Engineering, P.O. Box 208267, New Haven, CT 06520-8267

67. R. D. Rafler, 8906 Talbot Avenue, Silver Spring, MD 20910

68. Dr. Rodney Roberts, FAMU/FSU, College of Engineering, Department of Electrical Engineering, 2525 Pottsdamer Street, Tallahassee, FL 32310

69. Dr. Wes Snyder, Department of Radiology, Bowman Gray School of Medicine, N.C. Baptist Hospital School of Medicine, 300 S. Hawthorne Dr., WinstonSalem, NC 27103

70. Professor Mary F. Wheeler, Department of Mathematical Sciences, Rice University, P.O. Box 1892, Houston, TX 77251

71. Dr. Yong Zhou, 14616 NE 36th, Apt \#E-13, Bellevue, WA 98007

72. Office of Assistant Manager for Energy Research and Development, U.S. Department of Energy, Oak Ridge Operations Office, P.O. Box 2001, Oak Ridge, TN 37831-8600

73-74. Office of Scientific \& Technical Information, P. O. Box 62, Oak Ridge, TN 37830 